J. Austral. Math. Soc. 19 (Series B), (1976), 452-461.

\title{
A BOLTZMANN-TYPE EQUATION IN THE KINETIC THEORY OF VEHICULAR TRAFFIC
}

\author{
C. V. PAO \\ (Received 11 December 1975) \\ (Revised 30 June 1976)
}

\begin{abstract}
In the theory of vehicular traffic flow on a highway the traffic interaction process is often considered as a collision similar to the particles' interaction in the kinetic theory of gases. This concept leads to a Boltzmann-type nonlinear integro-differential equation which governs the traffic density function. The purpose of this paper is to present a constructive method for the determination of a solution for this type of equation under certain boundary and initial conditions. Our method is by successive approximation which yields existence of both global and local solutions of the problem.
\end{abstract}

\section{Introduction}

In the investigation of vehicular traffic flow on a highway Prigogine et al. [7-9] proposed a model for the traffic concentration using the kinetic theory of gases. In their theory, the rate of change of the traffic density function is governed by two kinds of processes called interaction and relaxation. This may be expressed as

$$
\frac{d f}{d t} \equiv \frac{\partial f}{\partial t}+v \frac{\partial f}{\partial x}=\left(\frac{\partial f}{\partial t}\right)_{\mathrm{int}}+\left(\frac{\partial f}{\partial t}\right)_{\mathrm{rel}}
$$

where $f \equiv f(t, x, v)$ is the density function at time $t$, position $x$ moving with velocity $v,(\partial f / \partial t)_{\mathrm{nt}}$ is the change of vehicular density due to traffic interactions and $(\partial f / \partial t)_{\text {rel }}$ is the so-called relaxation term. The physical meaning of the interaction process is that under not very dilute traffic conditions, a fast-moving vehicle has to slow down when it catches up with a slow-moving vehicle and cannot pass. On the other hand, the relaxation process enables a 
driver to speed up towards the desired speed when there is a chance to pass or when the traffic is very dilute. The desired speed depends on several factors such as the driver's wishes, the legal constraints, the vehicular characteristics and the road and weather conditions. Using the arguments usually made in the kinetic theory of gases Prigogine et al. (cf. [7] or [9], p. 23) established the following relations:

$$
\begin{aligned}
& \left(\frac{\partial f}{\partial t}\right)_{\mathrm{int}}=(1-p) f(t, x, v) \int_{v_{1}}^{v_{2}}\left(v^{\prime}-v\right) f\left(t, x, v^{\prime}\right) d v^{\prime} \\
& \left(\frac{\partial f}{\partial t}\right)_{\mathrm{rel}}=-\frac{1}{T_{0}}\left(f(t, x, v)-f_{0}(t, x, v)\right)
\end{aligned}
$$

where $p=p(t, x, v)$ is the probability of passing $(0 \leqq p \leqq 1), v_{1}, v_{2}$ are two fixed velocities with $0 \leqq v_{1}<v_{2}<\infty$ and $T_{0}>0$ is the relaxation time. The function $f_{0}$, which is called the desired speed distribution function, is the vehicular density that would be realized if the traffic is very dilute or there is no interaction between vehicles. This function is assumed in the form of

$$
f_{0}(t, x, v)=\int_{v_{1}}^{v_{2}} k\left(v, v^{\prime}\right) f\left(t, x, v^{\prime}\right) d v^{\prime},
$$

where $k\left(v, v^{\prime}\right)$ is a prescribed function (cf. [1], [9]). Under these conditions, Eq. (1.1) becomes the following nonlinear Boltzmann-type traffic equation

$$
\frac{\partial f}{\partial t}+v \frac{\partial f}{\partial x}+\frac{1}{T_{0}} f=J(t, x, v ; f) \quad\left(0<t \leqq T, 0<x \leqq l, v_{1} \leqq v \leqq v_{2}\right),
$$

where $l$ is the length of the highway and

$$
\begin{aligned}
J(t, x, v ; f)= & \frac{1}{T_{0}} \int_{v_{1}}^{v_{2}} k\left(v, v^{\prime}\right) f\left(t, x, v^{\prime}\right) d v^{\prime} \\
& +(1-p) f(t, x, v) \int_{v_{1}}^{v_{2}}\left(v^{\prime}-v\right) f\left(t, x, v^{\prime}\right) d v^{\prime}+q(t, x, v) .
\end{aligned}
$$

In the definition of $J(t, x, v ; f)$ we have introduced an inhomogeneous term $q$ which takes into account any other possible source such as the source from the boundary through a suitable transformation. Notice that the relaxation term given in (1.2) leads to an essential distinction between the traffic equation (1.3) and the classical Boltzmann equation.

In addition to Eq. (1.3) we consider the following boundary and initial conditions:

$$
\begin{array}{ll}
f(t, 0, v)=0 & \left(0 \leqq t \leqq T, v_{1} \leqq v \leqq v_{2}\right) \\
f(0, x, v)=\phi(x, v) & \left(0<x \leqq l, v_{1} \leqq v \leqq v_{2}\right) .
\end{array}
$$


The purpose of this paper is to show the existence and uniqueness of a solution for the initial boundary-value problem (1.3)-(1.5) and to give a direct method for the construction of the solution. It is to be noted that the consideration of the homogeneous boundary condition (1.4) is only for convenience since a non-homogeneous condition in the form of $f(t, 0, v)=$ $h(t, v)$ can be reduced to (1.4) through the transformation $f \rightarrow f-h$ (assuming that $h$ is continuous in $v$, continuously differentiable in $t$ and $h(0, v)=$ $\phi(0, v))$. In this situation, Eqns. (1.3) and (1.5) remain the same form except with some different functions $k, q, \phi$ and the coefficient of $f$.

The vehicular traffic flow equation (1.3) has also been discussed by a number of other investigators in the field of transportation (cf. [1-5]). The existence problem has recently been investigated by Belleni-Morante and Barone [1] using semi-group approach which leads to the existence of a local solution. In this paper we prove the existence of a global solution using the approach of [6] in the treatment of Boltzmann equation for neutron transport. The advantage of this approach is that it yields an explicit recursion formula for the construction of the solution. This formula involves only straightforward integration and thus our method gives both analytical results and computational significance.

\section{Successive approximations}

Throughout the paper, we assume that $q, k$ and $\phi$ are continuous functions of their respective arguments, $\phi$ is differentiable in $x$ and $\phi(0, v)=$ $q(t, 0, v)=0$. Let $D=[0, T] \times[0, l] \times\left[v_{1}, v_{2}\right]$ and let $C(D)$ be the linear space of all continuous functions $f(t, x, v)$ on $D$ with $f(t, 0, v)=0$ on $[0, T] \times\left[v_{1}, v_{2}\right]$. Define the usual maximum norm in $C(D)$ by

$$
\|f\|=\max \{|f(t, x, v)| ;(t, x, v) \in D\} .
$$

Then $C(D)$ is a Banach space. (In fact, $C(D)$ is a closed subspace of the Banach space of all continuous furtctions with the above norm.) For any preassigned constant $M>0$ we define a function $\tilde{J}(t, x, v ; f)$ by

$$
\tilde{J}(t, x, v ; f)=\left\{\begin{array}{ccc}
J(t, x, v ; f) & \text { if } & |f| \leqq M \\
J(t, x, v ; M) & \text { if } & f>M \\
J(t, x, v ;-M) & \text { if } & f<-M
\end{array}\right.
$$

Then we consider the following initial boundary-value problem:

$$
\left(\frac{\partial}{\partial t}+v \frac{\partial}{\partial x}\right) f+\frac{1}{T_{0}} f=\tilde{J}(t, x, v ; f) \quad\left(0<t \leqq T, 0<x \leqq l, v_{1} \leqq v \leqq v_{2}\right)
$$




$$
\begin{array}{ll}
f(t, 0, v)=0 & \left(0<t \leqq T, v_{1} \leqq v \leqq v_{2}\right) \\
f(0, x, v)=\phi(x, v) & \left(0<x \leqq l, v_{1} \leqq v \leqq v_{2}\right)
\end{array}
$$

where $((\partial / \partial t)+v(\partial / \partial x)) f$ is considered as the substantial derivative of $f$. The system (2.2)-(2.4), called a modified problem of (1.3)-(1.5), coincides with the original problem except with $J$ replaced by $\tilde{J}$. Hence if $\tilde{f}(t, x, v)$ is a solution of (2.2)-(2.4) then it is also a solution of (1.3)-(1.5) for as long as $|\tilde{f}(t, x, v)| \leqq M$. To investigate the existence of a solution for $(2.2)-(2.4)$ we make the transformation $f \rightarrow e^{-\lambda t} f$ to obtain the transformed system

$$
\begin{aligned}
\left(\frac{\partial}{\partial t}+\frac{\partial}{\partial x}\right) f+\left(\lambda+T_{0}^{-1}\right) f & =e^{-\lambda t} \bar{J}\left(t, x, v ; e^{\lambda t} f\right) \\
f(t, 0, v) & =0 \\
f(0, x, v) & =\phi(x, v)
\end{aligned}
$$

where $\lambda \geqq 0$ is a constant to be determined (see Theorem 1). We first show that the transformed problem (2.5)-(2.7) has a unique solution which can be constructed by successive approximation.

Let $f^{(0)}$ be any function in $C(D)$. Define a sequence $\left\{f^{(n)}\right\}$ successively from the linear system

$$
\begin{aligned}
& \left(\frac{\partial}{\partial t}+v \frac{\partial}{\partial x}\right) f^{(n)}+\left(\lambda+T_{0}^{-1}\right) f^{(n)}=e^{-\lambda t} \bar{J}\left(t, x, v ; e^{\lambda t} f^{(n-1)}\right) \\
& \left(0<t \leqq T, 0<x \leqq l, v_{1} \leqq v \leqq v_{2}\right) \\
& f^{(n)}(t, 0, v)=0 \quad\left(0<t \leqq T, v_{1} \leqq v \leqq v_{2}\right) \\
& f^{(n)}(0, x, v)=\phi(x, v) \quad\left(0<x \leqq l, v_{1} \leqq v \leqq v_{2}\right) \\
& n=1,2, \cdots
\end{aligned}
$$

The sequence $\left\{f^{(n)}\right\}$ is well-defined and is given by the recursion formula

$$
\begin{aligned}
& f^{(n)}(t, x, v)=e^{-\lambda_{r^{\prime}} \phi} \phi(x-v t, v) \\
& +e^{-\lambda_{0}{ }^{t}} \int_{0}^{t} e^{\tau / T_{0}} \tilde{J}\left(\tau, x-v(t-\tau), v ; e^{\lambda t} f^{(n-1)}(\tau, x-v(t-\tau), v)\right) d \tau \\
& n=1,2, \cdots
\end{aligned}
$$

where $\lambda_{0}=\lambda+T_{0}^{-1}$ (see Lemma 1). In obtaining the above formula we have set

$$
f(t, x, v)=q(t, x, v)=\phi(x, v)=0 \quad \text { when } \quad x<0 .
$$

Our main concern is to show that the sequence $\left\{f^{(n)}\right\}$ converges in $C(D)$ to a unique solution of the transformed problem (2.5)-(2.7). For this purpose, we define operators $A, \tilde{J}_{\lambda}$ by 


$$
\left.\begin{array}{cc}
A f=\left(\frac{\partial}{\partial t}+v \frac{\partial}{\partial x}\right) f+\lambda_{0} f & (f \in D(A)) \\
\left(\tilde{J}_{\lambda}(f)\right)(t, x, v)=e^{-\lambda t \tilde{J}\left(t, x, v ; e^{\lambda t} f(t, x, v)\right)} & (f \in C(D))
\end{array}\right\}
$$

where $D(A)$ is the domain of $A$ given by

$$
D(A)=\{f \in C(D) ; A f \in C(D) \text { and } f \text { satisfies (2.6),(2.7)\}. }
$$

Then the problem (2.5)-(2.7) is equivalent to the operator equation

$$
A f=\tilde{J}_{\lambda}(f) \quad(f \in D(A))
$$

in the Banach space $C(D)$ and the system (2.8) becomes

$$
A f^{(n)}=\tilde{J}_{\lambda}\left(f^{(n-1)}\right) \quad\left(f^{(n)} \in D(A)\right)
$$

The requirement of $f$ and $f^{(n)}$ in $D(A)$ is to insure that these functions satisfy the boundary and initial conditions (2.6), (2.7). Thus it suffices to show that the sequence $\left\{f^{(n)}\right\}$ determined from (2.14) converges to a unique solution of (2.13). To accomplish this, we derive the recursion formula (2.9) and establish some properties for the operators $A$ and $\tilde{J}_{\lambda}$ in the following lemmas.

LEMMA 1. For any $f^{(0)} \in C(D)$ the sequence $\left\{f^{(n)}\right\}$ in the system $(2.8)$ is in $D(A)$ and is given by the recursion formula (2.9).

Proof. Let $n$ be fixed and let $t=\tau, x^{\prime}=x-v t$. Then the first equation in (2.8) is equivalent to

$$
\begin{aligned}
\frac{d}{d \tau} f^{(n)}\left(\tau, x^{\prime}+\right. & v \tau, v)+\lambda_{0} f^{(n)}\left(\tau, x^{\prime}+v \tau, v\right) \\
& =e^{-\lambda \tau}\left[\tilde{J}\left(e^{\lambda \tau} f^{(n-1)}\right)\right]\left(\tau, x^{\prime}+v \tau, v\right)
\end{aligned}
$$

where $[\tilde{J}(f)](t, x, v)=\tilde{J}(t, x, v ; f)$. Multiplication by $e^{\lambda_{0} \tau}$ followed by integration from 0 to $t$ yields

$$
\begin{aligned}
e^{\lambda_{0} t} f^{(n)}\left(t, x^{\prime}\right. & +v t, v)-f^{(n)}\left(0, x^{\prime}, v\right) \\
= & \int_{0}^{t} e^{\left(\lambda_{1}-\lambda\right) \tau}\left[\tilde{J}\left(e^{\lambda \tau} f^{n-1}\right)\right]\left(\tau, x^{\prime}+v \tau, v\right) d \tau .
\end{aligned}
$$

Upon replacing $x^{\prime}$ by $x-v t$ and using the initial condition we obtain the formula (2.9). It is obvious that the function $f^{(n)}$ given by (2.9) satisfies the first and third equations in (2.8). Since by (2.10),

$$
\begin{aligned}
\phi(-v t, v) & =f^{(n-1)}(\tau,-v(t-\tau), v) \\
& =q(\tau,-v(t-\tau), v)=0 \quad \text { for } \quad t>\tau \geqq 0
\end{aligned}
$$


we see from the definition of $J$ and (2.9) that $f^{(n)}(t, 0, v)=0$. This shows that $f^{(n)}$ satisfies the boundary condition in (2.8). Therefore $f^{(n)} \in D(A)$ and is the desired solution of (2.8).

Lemma 2. The inverse operator $A^{-1}$ exists on $C(D)$ and

$$
\left\|A^{-1} g_{1}-A^{-1} g_{2}\right\| \leqq \lambda_{0}^{-1}\left\|g_{1}-g_{2}\right\| \quad\left(g_{1}, g_{2} \in C(D)\right) .
$$

Proof. We first show that $A^{-1}$ exists by establishing the inequality

$$
\left\|A f_{1}-A f_{2}\right\| \geqq \lambda_{0}\left\|f_{1}-f_{2}\right\| \quad\left(f_{1}, f_{2} \in D(A)\right) .
$$

It is obvious that (2.18) holds when $f_{1}=f_{2}$. For $f_{1} \neq f_{2}$ we set $f=f_{1}-f_{2}$ and let $z_{0}=\left(t_{0}, x_{0}, v_{0}\right) \in D$ such that $\|f\|=\left|f\left(t_{0}, x_{0}, v_{0}\right)\right|$. Then from $f(0, x, v)=$ $f(t, 0, v)=0$ we have $t_{0} \neq 0$ and $x_{0} \neq 0$. Since

$$
f\left(z_{0}\right)\left[\left(A f_{1}-A f_{2}\right)\left(z_{0}\right)\right]=\frac{1}{2}\left(\frac{\partial}{\partial t}+v_{0} \frac{\partial}{\partial x}\right) f^{2}\left(z_{0}\right)+\lambda_{0} f^{2}\left(z_{0}\right)
$$

and since $f^{2}\left(z_{0}\right)$ is a positive maximum in $D$ we see that the first term on the right side of (2.19) is either zero or positive. This implies that

$$
\|f\|\left\|A f_{1}-A f_{2}\right\| \geqq f\left(z_{0}\right)\left[\left(A f_{1}-A f_{2}\right)\left(z_{0}\right)\right] \geqq \lambda_{0}\|f\|^{2}
$$

and thus (2.18) follows. Now for any $g_{1}, g_{2} \in C(D)$ the proof of Lemma 1 insures that there exist $f_{1}, f_{2} \in D(A)$ such that $A f_{1}=g_{1}, A f_{2}=g_{2}$. Then by (2.18) we obtain (2.17) immediately.

We next give some estimates for the function $\tilde{J}$. For convenience, we set

$$
\begin{aligned}
& k_{1}=\sup \left\{\int_{v_{1}}^{v_{2}}\left|k\left(v, v^{\prime}\right)\right| d v^{\prime} ; v_{1} \leqq v \leqq v_{2}\right\} \\
& k_{2}=\sup \left\{\int_{v_{1}}^{v_{2}}\left|v^{\prime}-v\right| d v^{\prime} ; v_{1} \leqq v \leqq v_{2}\right\} \\
& k_{M}=T_{0}^{-1} k_{1}+2 k_{2} M .
\end{aligned}
$$

LEMma 3. For any $f_{1}, f_{2} \in C(D)$ and any fixed point $z \equiv(t, x, v)$ in $D$,

$$
\left|\tilde{J}\left(z, e^{\lambda t} f_{1}(z)\right)-\tilde{J}\left(z, e^{\lambda t} f_{2}(z)\right)\right| \leqq K_{M} e^{\lambda t}\left\|f_{i}-f_{2}\right\| .
$$

Proof. For each $i=1,2$, define functions $\tilde{f}$, by

$$
\tilde{f}_{i}\left(t, x, v^{\prime}\right)=\left\{\begin{array}{ccc}
M e^{-\lambda t} & \text { when } & f_{i}\left(t, x, v^{\prime}\right)>M e^{-\lambda t} \\
f_{i}\left(t, x, v^{\prime}\right) & \text { when } & \left|f_{i}\left(t, x, v^{\prime}\right)\right| \leqq M e^{-\lambda t} \\
-M e^{-\lambda t} & \text { when } & f_{i}\left(t, x, v^{\prime}\right)<-M e^{-\lambda t}
\end{array}\right.
$$

Then by the definition of $\tilde{J}$, 


$$
\begin{aligned}
\tilde{J}\left(z, e^{\lambda \prime} f_{2}(z)\right)-\tilde{J}\left(z, e^{\lambda \prime} f_{1}(z)\right) \\
=\frac{1}{T_{0}} \int_{v_{1}}^{\nu_{2}} k\left(v, v^{\prime}\right)\left[e^{\lambda t} \tilde{f}_{2}\left(t, x, v^{\prime}\right)-e^{\lambda \prime} \tilde{f}_{1}\left(t, x, v^{\prime}\right)\right] d v^{\prime} \\
\quad+(1-p) e^{\lambda \prime} \tilde{f}_{2}(z) \int_{v_{1}}^{v_{2}}\left(v^{\prime}-v\right)\left[e^{\lambda \prime} \tilde{f}_{2}\left(t, x, v^{\prime}\right)-e^{\lambda t} \tilde{f}_{1}\left(t, x, v^{\prime}\right)\right] d v^{\prime} \\
+(1-p)\left[e^{\lambda \prime} \tilde{f}_{2}(z)-e^{\lambda t} \tilde{f}_{1}(z)\right] \int_{v_{1}}^{v_{2}}\left(v^{\prime}-v\right) e^{\lambda t} \tilde{f}_{1}\left(t, x, v^{\prime}\right) d v^{\prime} .
\end{aligned}
$$

We first show that for any $v^{\prime} \in\left[v_{1}, v_{2}\right]$,

$$
\left|\tilde{f}_{2}\left(t, x, v^{\prime}\right)-\tilde{f}_{1}\left(t, x, v^{\prime}\right)\right| \leqq\left|f_{2}\left(t, x, v^{\prime}\right)-f_{1}\left(t, x, v^{\prime}\right)\right|
$$

The above inequality is clearly satisfied if for both $i=1,2$, either $\left|f_{1}\left(t, x, v^{\prime}\right)\right| \leqq$ $M e^{-\lambda_{t}}$ or $\left|f_{1}\left(t, x, v^{\prime}\right)\right|>M e^{-\lambda t}$. Thus it suffices to show the cases

(i) $f_{2}\left(t, x, v^{\prime}\right)>M e^{-\lambda t}, f_{1}\left(t, x, v^{\prime}\right) \leqq M e^{-\lambda t}$ and

(ii) $-M e^{-\lambda t} \leqq f_{2}\left(t, x, v^{\prime}\right) \leqq M e^{-\lambda t}, f_{1}\left(t, x, v^{\prime}\right)<-M e^{-\lambda t}$.

Let $z^{\prime}=\left(t, x, v^{\prime}\right)$. Since by the definition of $\tilde{f}_{1}\left(z^{\prime}\right), \bar{f}_{2}\left(z^{\prime}\right) \leqq f_{2}\left(z^{\prime}\right)$ when $f_{2}\left(z^{\prime}\right) \geqq$ $-M e^{-\lambda t}$. We see that for both cases (i) and (ii),

$$
0<\tilde{f}_{2}\left(z^{\prime}\right)-\tilde{f}_{1}\left(z^{\prime}\right) \leqq f_{2}\left(z^{\prime}\right)-\tilde{f}_{1}\left(z^{\prime}\right) \text {. }
$$

But $\tilde{f}_{1}\left(z^{\prime}\right) \geqq f_{1}\left(z^{\prime}\right)$ when $f_{1}\left(z^{\prime}\right) \leqq M e^{-\lambda t}$ we obtain

$$
0<\tilde{f}_{2}\left(z^{\prime}\right)-\tilde{f}_{1}\left(z^{\prime}\right) \leqq f_{2}\left(z^{\prime}\right)-f_{1}\left(z^{\prime}\right) .
$$

This proves the inequality (2.21). In view of this inequality and the fact that $\left|\tilde{f}_{r}\left(z^{\prime}\right)\right| \leqq M e^{-\lambda t}$ we have

$$
\begin{aligned}
& \left|\tilde{J}\left(z, e^{\lambda \prime} f_{2}(z)\right)-\tilde{J}\left(z, e^{\lambda t} f_{1}(z)\right)\right| \\
& \quad \leqq\left(e^{\lambda t} / T_{0}\right) \int_{v_{i}}^{v_{2}}\left|k\left(v, v^{\prime}\right)\right|\left|f_{2}\left(t, x, v^{\prime}\right)-f_{1}\left(t, x, v^{\prime}\right)\right| d v^{\prime} \\
& \quad+(1-p) M e^{\lambda t} \int_{v_{1}}^{v_{2}}\left|v^{\prime}-v\right|\left|f_{2}\left(t, x, v^{\prime}\right)-f_{1}\left(t, x, v^{\prime}\right)\right| d v^{\prime} \\
& \quad+(1-p) M e^{\lambda t}\left|f_{2}(z)-f_{1}(z)\right| \int_{v_{1}}^{v_{2}}\left|v^{\prime}-v\right| d v^{\prime}
\end{aligned}
$$

Since the first term on the right side is bounded by $\left(k_{1} / T_{0}\right) e^{\wedge t}\left\|f_{2}-f_{1}\right\|$ and the second and third term are both bounded by $(1-p) M k_{2} e^{\lambda t}\left\|f_{2}-f_{1}\right\|$ we conclude that the right side of the above inequality is bounded by

$$
\left(k_{1} e^{\lambda \prime} / T_{0}\right)\left\|f_{1}-f_{2}\right\|+2\left((1-p(z)) M k_{2} e^{\lambda \prime}\right)\left\|f_{1}-f_{2}\right\| .
$$

Since $0 \leqq p \leqq 1$ the inequality (2.20) follows immediately from (2.22).

We now prove the existence theorem for the modified problem. 
THEOREM 1. Let $\lambda$ be any constant satisfying $\lambda \geqq K_{m}$. Then with any $f^{(0)} \in C(D)$ the sequence $\left\{f^{(n)}\right\}$ given by (2.9) converges uniformly in $D$ to a unique solution $\tilde{f}$ of the problem (2.5)-(2.7). Furthermore,

$$
\left\|f^{(n)}-\bar{f}\right\| \leqq \frac{K_{M}}{\lambda_{0}-K_{M}}\left(\frac{K_{M}}{\lambda_{0}}\right)^{n-1}\left\|f^{(1)}-f^{(0)}\right\|, \quad n=1,2, \cdots,
$$

where $\lambda_{0}=\lambda+T_{0}^{-1}$.

Proof. It suffices to show that the sequence $\left\{f^{(n)}\right\}$ determined from (2.14) converges in $C(D)$ to a unique solution of (2.13). Since for each $n=1,2, \cdots, \tilde{J}_{\lambda}\left(f^{(n-1)}\right)$ is in $C(D)$ and by Lemma $2 A^{-1}$ exists on $C(D)$ we may write $(2.14)$ as

$$
f^{(n)}=A^{-1} \tilde{J}_{\lambda}\left(f^{(n-1)}\right) \quad n=1,2, \cdots
$$

We show that the operator $A^{-1} \tilde{J}_{\lambda}$ is a contraction mapping on $C(D)$. Let $f_{1}, f_{2} \in C(D)$ and let $z$ be any point in $D$. By the definition of $\tilde{J}_{\lambda}(f)$ and (2.20) we have

$$
\begin{aligned}
\left|\left(\tilde{J}_{\lambda}\left(f_{1}\right)\right)(z)-\left(\tilde{J}_{\lambda}\left(f_{2}\right)\right)(z)\right| & =e^{-\lambda \iota}\left|\tilde{J}\left(z, e^{\lambda \prime} f_{1}(z)\right)-\tilde{J}\left(z, e^{\lambda \prime} f_{2}(z)\right)\right| \\
& \leqq K_{M}\left\|f_{1}-f_{2}\right\| .
\end{aligned}
$$

The above inequality implies that

$$
\left\|\tilde{J}_{\lambda}\left(f_{1}\right)-\tilde{J}_{\lambda}\left(f_{2}\right)\right\| \leqq K_{M}\left\|f_{1}-f_{2}\right\| \quad\left(f_{1}, f_{2} \in C(D)\right) .
$$

In view of Lemma 2 ,

$$
\begin{aligned}
&\left\|A^{-1} \tilde{J}_{\lambda}\left(f_{1}\right)-A^{-1} \tilde{J}_{\lambda}\left(f_{2}\right)\right\| \\
& \quad \leqq \lambda_{0}^{-1}\left\|\tilde{J}_{\lambda}\left(f_{1}\right)-\tilde{J}_{\lambda}\left(f_{2}\right)\right\| \leqq\left(K_{M} / \lambda_{0}\right)\left\|f_{1}-f_{2}\right\| .
\end{aligned}
$$

Hence the choice of $\lambda \geqq K_{M}$ insures that $A^{-1} \bar{J}_{\lambda}$ is a contraction on $C(D)$ with a contraction constant $\left(K_{M} / \lambda_{0}\right)<1$. It follows from the contraction property of $A^{-1} \tilde{J}_{\lambda}$ that the sequence $\left\{f^{(n)}\right\}$ given by (2.24) converges in $C(D)$ to a unique solution $\bar{f}$ of the equation

$$
f=A^{-1} \tilde{J}_{\lambda}(f)
$$

and satisfies the error estimate (2.23). This implies that $\bar{f} \in D(A)$ and $A \tilde{f}=\tilde{J}_{\lambda}(\tilde{f})$, that is, $\tilde{f}$ is the desired solution of (2.13). Since (2.13) is equivalent to (2.5)-(2.7) and (2.14) is equivalent to (2.8) the conclusions in the theorem follow immediately. This proves the theorem.

By letting $F^{(n)}(t, x, v)=e^{\lambda t} f^{(n)}(t, x, v)$ for each $n$ then the formula (2.9) becomes 


$$
\begin{aligned}
& F^{(n)}(t, x, v)=e^{-\left(t / r_{\alpha}\right)} \phi(x-v t, v) \\
& +\int_{0}^{t} e^{-(1-t) / T_{0}} \tilde{J}\left(\tau, x-v(t-\tau), v ; F^{(n-1)}(\tau, x-v(t-\tau), v)\right) d \tau \\
& n=1,2, \cdots
\end{aligned}
$$

Since the convergence of $\left\{f^{(n)}\right\}$ to $\tilde{f}$ implies the convergence of $\left\{F^{(n)}\right\}$ to $f \equiv e^{\lambda t} \tilde{f}$ and since the problem (2.5)-(2.7) reduces to the problem (2.2)-(2.4) with the substitution of $f$ by $e^{\lambda} f$ we obtain the following

THEOREM 2. For any $F^{(0)} \in C(D)$ the sequence $\left\{F^{(n)}\right\}$ given by (2.29) converges uniformly in $D$ to a unique solution $f$ of the problem (2.2)-(2.4).

REMARK. In Theorem 1 there is an error estimate for the approximations $f^{(n)}$. However, we do not have a direct error estimate for $F^{(n)}$.

In view of the definition of $\tilde{J}$ the solution $f$ of the modified problem (2.2)-(2.4) is the solution of the original problem (1.3)-(1.5) for at least $|f(t, x, v)| \leqq M$. Since the value of $M$ can be preassigned we see that for each $M$ greater than the maximum of the initial function $\phi(x, v)$ there exists a $T_{M}>0$ such that $|f(t, x, v)| \leqq M$ for $0 \leqq t \leqq T_{M}, 0 \leqq x \leqq l, v_{1} \leqq v \leqq v_{2}$. This observation leads to the following conclusion for the original problem.

THEOREM 3. The problem (1.3)-(1.5) has a unique solution $f(t, x, v)$ which is either bounded on the whole domain $D$ or there exists finite $T^{*} \leqq T$ such that $f(t, x, v)$ exists on $\left[0, T^{*}\right)$ and is unbounded at some point $(x, v)$ when $t \rightarrow T^{*}$. In any case, the solution $f$ can be obtained from the recursion formula (2.29) with $J$ replaced by $\tilde{J}$.

Proof. The existence of the solution $f$ as well as its construction follow from Theorem 2. Now if there is an $M$ such that the solution $\tilde{f}$ obtained from Theorem 2 is bounded by $M$ then $\tilde{f}$ is the desired solution of the original problem. If, on the other hand, $\|\tilde{f}\|>M^{\prime}$ for every value of $M^{\prime}$ in the definition of $\tilde{J}$ then $\tilde{f}$ is the solution of (1.3)-(1.5) only for $|\tilde{f}(t, x, v)| \leqq M$. In this case the original solution $f$ must be unbounded. For it were bounded (say, by $M_{0}$ ) then by defining $\tilde{J}$ with $M=M_{0}$ we have $\tilde{J}(f)=J(f)$ and thus the solution $\tilde{f}$ coincides with $f$. Notice that in any case, the function $\tilde{f}$ is bounded and can be determined from (2.29).

The result of Theorem 3 gives a direct method for the calculation of approximate solutions to the problem (1.3)-(1.5) even if the solution is unbounded. It is to be noted that in the process of calculating the approximate solutions $F^{(n)}$, the modified function $\bar{J}$ should be used in the recursion formula (2.29) throughout the calculation since it is not known whether the sequence $\left\{F^{(n)}\right\}$ converges if the original function $J$ is used. Since the solution $\bar{f}$ of the 
modified problem can always be constructed through the recursion formula (2.29), if we assign $M$ very large then $\tilde{f}$ is the desired solution of the original problem for any finite interval $[0, T]$ unless the original solution grows unbounded in a finite time $T^{*} \leqq T$ (which is unlikely from physical point of view). This procedure has some practical implications since the solution of (1.3)-(1.5) can be constructed from (2.29) without the usual process of continuation of a solution which requires a new initial function in each step of continuation. On the other hand, it is easily seen from the proof of Lemma 3 that Theorems 1 to 3 remain true if the function $k\left(v, v^{\prime}\right)$ is replaced by a continuous function $k\left(t, x, v, v^{\prime}\right)$ which may depend on $t$ and $x$. In fact, the above method can be used for more general type of desired distribution function $f_{0}$.

\section{References}

[1] A. Belleni-Morante and E. Barone, 'Nonlınear kinetic theory of vehicular traffic', J. Math. Anal. Appl. 47(1974), 443-457.

[2] A. V. Gafarian, P. Munjal and J. Pahl, 'An experimental validation of the Boltzmann-type statistical models for multi-lane traffic flow', Transportation Res., 5 (1971), 211-224.

[3] D. C. Gazis, 'Mathematical theory of automobile traffic', Science, 157 (1967), 273-281.

[4] F. A Haight, Mathematical Theory for Traffic Flow, Academic Press, New York, 1963.

[5] P. Munjal and J. Pahl, "An analysis of the Boltzmann-type statistical models for multi-lane traffic flow', Transportation Res, 3 (1969), 151-163.

[6] C. V. Pao, 'Solution of a nonlinear Boltzmann equation for neutron transport in $L$ ' space', Arch. Rat. Mech. Anal, 50 (1973), 290-302.

[7] I. Prigogine and F. C. Andrews, 'A Boltzmann-like approach for traffic flow', Ops. Res., 8 (1960), 789-797.

[8] I. Prigogine, 'A Boltzmann-like approach to the statistical theory of traffic flow', Proc. Symposium on Theory of Traffic Flow, Edited by R. Herman (1961), 158-164, Elsevier, Amsterdam.

$[9]$ I. Prigogine and R. Herman, Kinetic Theory of Vehicular Traffic, American Elsevier, New York, 1971 .

North Carolina State University, Raleigh, North Carolina 27607, U.S.A. 